\title{
Long non-coding RNA H19 regulates Bcl-2, Bax and phospholipid hydroperoxide glutathione peroxidase expression in spontaneous abortion
}

\author{
RU-XIA BAI and ZOU-YING TANG
}

Department of Reproductive Medicine, The First People's Hospital of Yunnan Province, Kunming, Yunnan 650032, P.R. China

Received November 12, 2019; Accepted June 5, 2020

DOI: $10.3892 /$ etm. 2020.9473

\begin{abstract}
Spontaneous abortion (SA) is the most frequently occurring pregnancy disorder and is a serious threat to women's health. Identifying novel risk factors and the molecular mechanisms underlying SA are important. The present study reported that the RNA expression levels of long non-coding RNA H19 were lower in SA group compared with those in the control group, and the expression of Bax was increased and levels of $B c l-2$ and phospholipid hydroperoxide glutathione peroxidase (GPX4) were decreased in SA group at both the mRNA and protein levels. H19 expression was positively correlated with Bcl-2 and GPX4 expression and negatively linked with Bax levels. It was demonstrated that silencing H19 downregulated $B c l-2$ and GPX4 expression and upregulated Bax expression at both the mRNA and protein levels in HTR-8/SVneo trophoblast cells. In conclusion, the present findings suggested that H19 has important roles in SA by promoting apoptosis and ferroptosis.
\end{abstract}

\section{Introduction}

The incidence of spontaneous abortion (SA) is $10-15 \%$ in clinical pregnancies globally (1). The definition of SA is clinically confirmed pregnancy loss within 24 weeks of pregnancy $(2,3)$. The etiology of pregnancy loss is complicated, and genetic factors such as chromosomal abnormalities and single gene disorders, endocrinological factors such as diabetes mellitus and thyroid disease, environmental factors such as alcohol and tobacco and infectious factors such as viral and bacterial factors are all the risk factors of SA $(4,5)$. However, there may still be other unknown factors contributing to SA, therefore further investigation is needed.

Correspondence to: Dr Zou-Ying Tang, Department of Reproductive Medicine, The First People's Hospital of Yunnan Province, 157 Jinbi Road, Xishan, Kunming, Yunnan 650032, P.R. China

E-mail: 84442034@qq.com

Key words: long non-coding RNA H19, Bcl-2, Bax, phospholipid hydroperoxide glutathione peroxidase, spontaneous abortion
The cellular mechanisms underlying recurrent abortion are the proliferation and apoptosis of cytotrophoblasts and human decidual cells (6). Zhang et al (7) reported that microRNA (miR)-184 is highly expressed in decidual stromal cells, decidual immune cells and peripheral blood of patients who experience recurrent spontaneous abortion (RSA). Mechanically, miR-184 promotes apoptosis and inhibits the proliferation of trophoblast cells by targeting and regulating WIG1 (7). miR-520 also enhances the apoptosis of trophoblast cells by targeting poly (ADP-ribose) polymerase (PARP) 1 in human-trophoblast-derived HTR-8/SVneo cells (8). Ferroptosis is form of programed cell death that is different from apoptosis both in morphology and biochemical levels such as caspase-3 activation, and is characterized by the accumulation of reactive oxygen species (ROS) as a result of iron accumulation and lipid peroxidation (9). Ferroptosis is involved in several diseases, such as ischemia/reperfusion-induced organ injury, stroke and cancer, and ferroptosis inhibition is effective in treating ischemia/reperfusion-induced organ injury and stroke in a number of experimental models in vivo (10-12). However, whether ferroptosis is involved in the process of SA is still unknown.

Long non-coding RNAs (lncRNAs), which are $>200$ nucleotides in length, have important roles in a number of cellular processes, including posttranslational regulation and carcinogenesis $(13,14)$. H19 is a lncRNA that is $2.3 \mathrm{~kb}$ long and is primarily located in cytoplasm. During embryonic development, H19 is highly expressed; however, after birth, the expression of H19 is decreased (15). Most studies investigating H19 have focused on its role in carcinogenesis, including tumor growth and metastasis $(16,17)$, and although several studies have analyzed the expression and methylation status of H19 in SA $(18,19)$, whether $\mathrm{H} 19$ regulates apoptosis and ferroptosis in SA is unknown.

The present study analyzed the expression levels of $H 19$ and the apoptosis- and ferroptosis-associated genes $\mathrm{Bcl}-2$, $B a x$ and phospholipid hydroperoxide glutathione peroxidase $(G P X 4)$ in SA tissues. Importantly, the current study also further evaluated the regulatory correlations between $H 19$ and $B c l / G P X 4 / B a x$ both at the mRNA and protein levels.

\section{Materials and methods}

Patient sample collection. The placental villi tissues from patients with SA (SA group) and clinically normal pregnancies 
terminated for non-medical reasons (control group) were collected. The SA group consisted of 30 women aged 21-38 years. The criteria for inclusion in SA group were as follows: i) Normal chromosome number and structure of both the mother and father, ii) no thyroid dysfunction, diabetes and other systemic diseases, iii) no reproductive endocrine disease, iv) no deformity of genital tract and uterus and v) the activity and quality of sperm of the father were normal. The control group included 30 women aged 21-38 years who requested termination due to an unplanned pregnancy. The criteria for control group were as follows: i) No vaginal bleeding, ii) no abdominal pain. All women in these two groups were between gestational weeks six to nine. The present study was approved by The Medical Ethics Committee of the First People's Hospital of Yunnan Province (approval no. 2018-012, Kunming, China) and written informed consent was provided by each patient.

Cell culture. HTR-8/SVneo cells (henceforth referred to as HTR-8) were purchased from the American Type Culture Collection and cultured in RPMI-1640 medium (Gibco; Thermo Fisher Scientific, Inc.) supplemented with $10 \%$ fetal bovine serum (Gibco; Thermo Fisher Scientific, Inc.), $100 \mathrm{U} / \mathrm{ml}$ penicillin and $100 \mu \mathrm{g} / \mathrm{ml}$ in $\mathrm{CO}_{2}$ at $37^{\circ} \mathrm{C}$.

Cell transfection. A H19 knockdown lentiviral vector (pGLVU6-puro-H19-shRNA) and negative vector (non-targeting control, pGLVU6-puro-NC-shRNA) were constructed by Shanghai GenePharma Co., Ltd. $3 \mu \mathrm{g} \mathrm{H} 19$ knockdown lentiviral vector/negative vector were co-transfected with $2.5 \mu \mathrm{g}$ pMD2.G and $7.5 \mu \mathrm{g}$ psPAX2 plasmids into $293 \mathrm{~T}$ cells $\left(75 \mathrm{~cm}^{2}\right.$ flasks) for lentivirus packaging with Lipofectamine 2000 reagent (Thermo Fisher Scientific, Inc.). The sequences are as follows: H19 short hairpin (sh)RNA, 5'-CCGGCAGCCTTCAAGCATTCCATTACTCGAGTTTTT G-3'; NC shRNA, 5'-CCGGTTCTCCGAACGTGTCACGTT TTTTG-3'. Lentivirus infection was applied to HTR-8 cells with 50-60\% confluence. Reverse transcription-quantitative (RT-q)PCR was used to examine the efficiency of H19 knockdown after $72 \mathrm{~h}$ of infection.

$R T$ - $q P C R$. TRIzol $^{\circledR}$ reagent (Invitrogen; Thermo Fisher Scientific, Inc.) was used to isolate total RNA of tissues and cells according to the protocol of the manufacturer. A QuantiTect Reverse Transcription kit (Qiagen China Co., Ltd.) was used to synthesize cDNA by incubating at $42^{\circ} \mathrm{C}$ for $15 \mathrm{~min}$. H19, Bcl-2, Bax and GPX4 levels were detected by using a SYBR Green RT-qPCR assay kit (Takara Biotechnology Co., Ltd.), and GAPDH was used as the endogenous control. The PCR program was as follows: Stage $1,95^{\circ} \mathrm{C}$ for $30 \mathrm{sec}$; stage 2, $95^{\circ} \mathrm{C}$ for $5 \mathrm{sec}$ and $60^{\circ} \mathrm{C}$ for $34 \mathrm{sec}$, for 40 cycles. The primer sequences were as follows: Bcl-2, forward, 5'-GGTGGGGTC ATGTGTGTGG-3' and reverse, 5'-CGGTTCAGGTACTCA GTCATCC-3'; Bax, forward, 5'-CCCGAGAGGTCTTTTTCC GAG-3' and reverse, 5'-CCAGCCCATGATGGTTCTGAT-3'; GPX4, forward, 5'-GAGGCAAGACCGAAGTAAACTAC-3' and reverse, 5'-CCGAACTGGTTACACGGGAA-3'; H19, forward, 5'-CGTGACAAGCAGGACATGACA-3' and reverse, 5'-CCATAGTGTGCCGACTCCG-3'; GAPDH, forward, 5'-GGAGCGAGATCCCTCCAAAAT-3' and reverse, 5'-GGC
TGTTGTCATACTTCTCATGG-3'. Quantitative measurements were evaluated using the $2^{-\Delta \Delta C q}$ method (20). The mRNA levels of H19, Bcl-2, Bax and GPX4 in shCON group were normalized to 1 .

Western blotting. HTR-8 cells were collected and lysed using RIPA lysis buffer (Beyotime Institute of Biotechnology) for $25 \mathrm{~min}$ on ice. Protein concentrations were evaluated using the BCA method. Equal amounts of proteins (7-10 $\mu \mathrm{g}$ per sample) were loaded per lane on a $12 \%$ gel, resolved using SDS-PAGE and subsequently transferred to PVDF membranes. Then the membranes were blocked with 5\% non-fat milk for $1 \mathrm{~h}$ at the room temperature. The membranes were treated with primary antibodies overnight at $4^{\circ} \mathrm{C}$. Next, the membranes were treated with the secondary antibodies for $1 \mathrm{~h}$ at room temperature. The protein signals were detected using ECL Detection Reagent (Beijing Solarbio Science \& Technology Co., Ltd.). The primary antibodies including anti-Bcl-2 (1:1,000; cat. no. 15071), anti-Bax (1:1,000; cat. no. 5023) and anti- $\beta$-actin (1:5,000; cat. no. 3700) antibodies were purchased from Cell Signaling Technology, Inc., and anti-GPX4 (1:1,000; cat. no. ab125066) was purchased from Abcam. The secondary antibodies including anti-mouse IgG, HRP-linked antibody (1:3,000; cat. no. 7076) and anti-rabbit IgG, HRP-linked antibody (1:3,000; cat. no. 7074) were purchased from Cell Signaling Technology, Inc. $\beta$-actin was used as the internal control.

Cell viabilityanalysis.AfterH19shRNAinfectionfor48h,HTR-8 cells were seeded in 96-well plates. The cells were then divided into six groups, the shCON, shH19, shCON+Z-VAD-FMK, shH19+Z-VAD-FMK, shCON+Ferrostatin-1 and the shH19+Ferrostatin-1 group. After treatment for $48 \mathrm{~h}$, cell viability was examined by using the Cell Counting Kit- 8 method as previously described (21). Z-VAD-FMK (Selleck Chemicals) was a strong apoptosis inhibitor, and ferrostatin-1 (Selleck Chemicals) was a ferroptosis inhibitor which could depress the cellular lipid peroxidation (22).

Statistical analysis. Data are presented as the mean \pm standard deviation. Statistical analysis was performed using SPSS version 19.0 software (IBM, Corp.). Data were analyzed using a two-tailed unpaired Student's t-test (two groups) or analysis of variance (ANOVA) with Tukey's post hoc test for multiple comparisons. The correlation between $\mathrm{Bax}, \mathrm{Bcl}-2$ and GPX4 mRNA expression with $\mathrm{H} 19$ levels was analyzed using Pearson's correlation. $\mathrm{P}<0.05$ was considered to indicate a statistically significant difference. All in vitro experiments were repeated in triplicate.

\section{Results}

Expression levels of $\mathrm{H} 19$ and apoptosis- and ferroptosisassociated genes in the SA group. Sixty patients were included in the present study, including 30 cases in the SA group and 30 cases in the control group. RT-qPCR was used to detect the RNA expression levels of $H 19, B c l-2, B a x$ and $G P X 4$. The results showed that the RNA expression levels of $H 19$ were significantly lower in SA group compared with those in the control group (Fig. 1A). It was further found that the mRNA expression of anti-apoptosis gene $\mathrm{Bcl}-2$ was significantly 

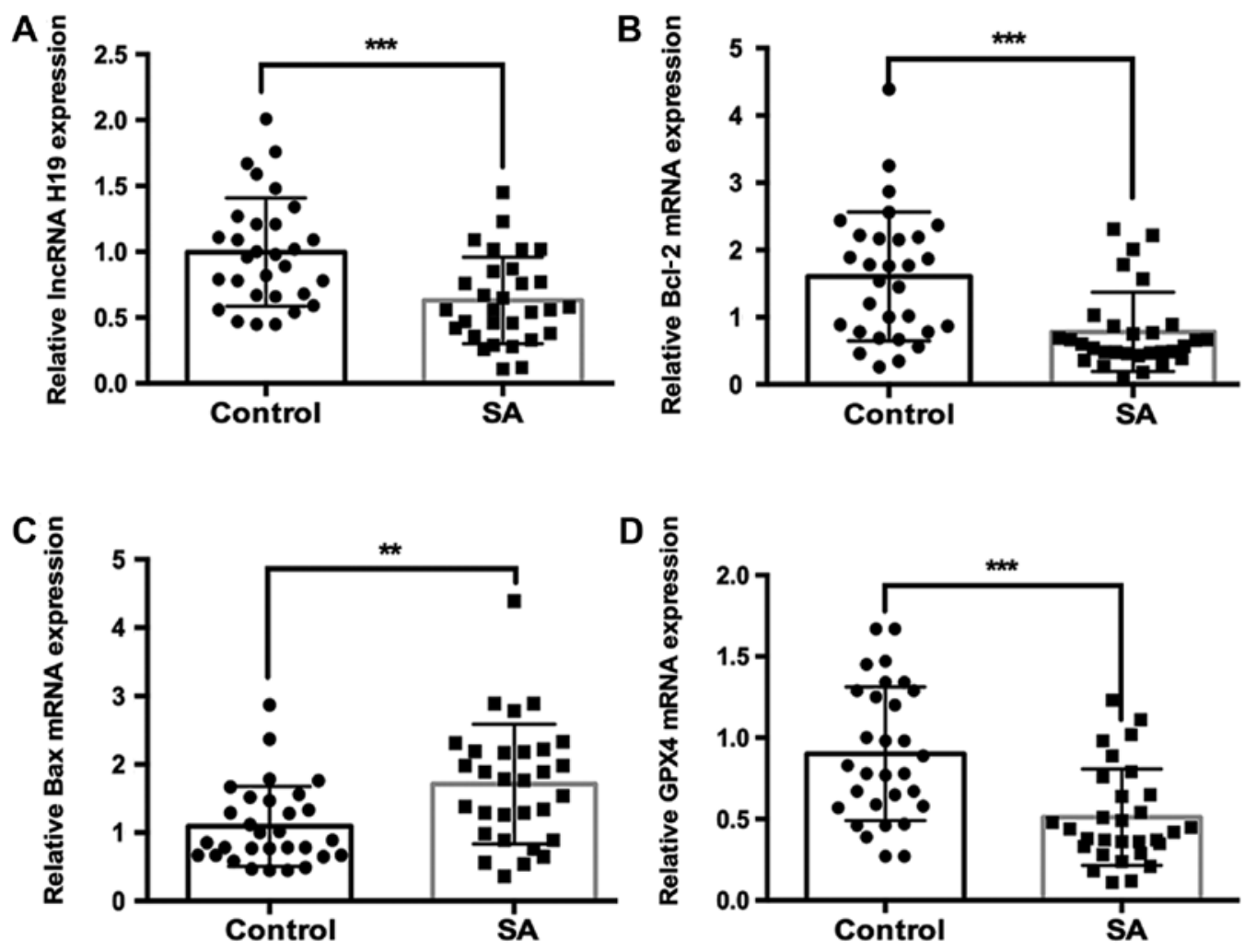

Figure 1. mRNA expression of $H 19, B c l-2, B a x$ and $G P X 4$ in SA. (A-D) Reverse transcription-quantitative PCR was used to detect the expression of $H 19, B c l$-2, Bax and GPX4 in the SA group $(\mathrm{n}=30)$ and control group $(\mathrm{n}=30) .{ }^{* *} \mathrm{P}<0.01$ and ${ }^{* * *} \mathrm{P}<0.001$ vs. control. SA, spontaneous abortion; lncRNA, long non-coding RNA; GPX4, phospholipid hydroperoxide glutathione peroxidase. Circle, expression data of each control sample; square, expression data of each SA sample.
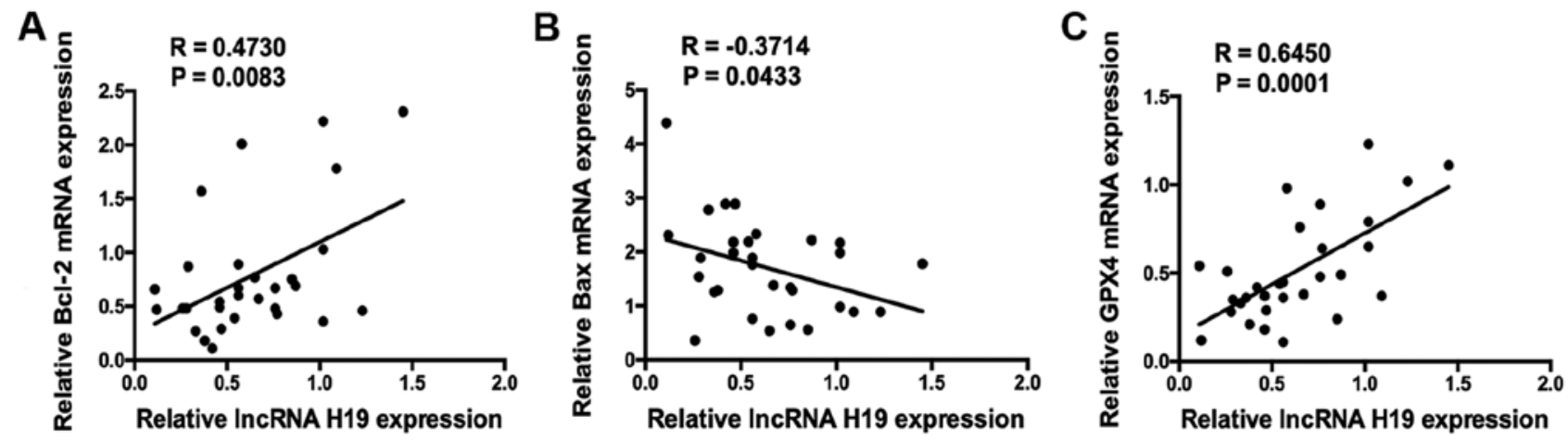

Figure 2. Correlation between the expression of $\mathrm{H} 19$ and (A) Bcl-2, (B) Bax and (C) GPX4 in the SA group. IncRNA, long non-coding RNA; GPX4, phospholipid hydroperoxide glutathione peroxidase. Circle, expression data of each SA sample. SA, spontaneous abortion.

lower, and the mRNA expression of proapoptotic gene Bax was significantly higher in SA group compared with those in control group (Fig. 1B and C). Notably, the ferroptosis-associated gene GPX4 was also significantly downregulated in SA group compared with the control group (Fig. 1D).

Correlation analysis was applied to evaluate relationship between the RNA expression levels of $\mathrm{H} 19$ and apoptosisand ferroptosis-associated genes. IncRNA H19 expression was significantly positively correlated with $\mathrm{Bcl}-2$ expression $(\mathrm{R}=0.4730, \mathrm{P}=0.0083)$, and significantly negatively correlated with Bax expression ( $\mathrm{R}=-0.3714, \mathrm{P}=0.0433$; Fig. $2 \mathrm{~A}$ and $\mathrm{B})$. Notably, it was also demonstrated that $H 19$ expression was significantly positively correlated with GPX4 expression $(\mathrm{R}=0.6450, \mathrm{P}=0.0001$; Fig. 2C).
Western blotting was used to evaluate the expression of apoptosis- and ferroptosis-associated proteins. The protein levels of Bax were higher in SA group, and Bcl-2 levels were lower in SA group compared those in the control group. In addition, GPX4 expression was downregulated in SA group compared with the control group (Fig. 3).

Silencing H19 downregulates Bcl-2 and GPX4 and upregulates Bax in HTR-8 cells. HTR-8 cells were transfected with the shH19 lentiviral construct, and the results showed that the expression of $H 19$ was significantly decreased by $\sim 80 \%$ (Fig. 4A). Then, the expressions of $B c l-2, B a x$ and GPX4 were evaluated using RT-qPCR and western blotting. The results showed that silencing H19 significantly reduced expression 


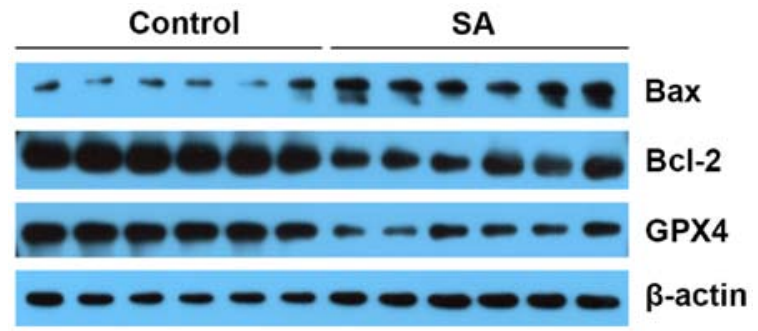

Figure 3. Protein expression of Bcl-2, Bax and GPX4 in SA group compared with the control group as analyzed using western blotting. SA, spontaneous abortion; GPX4, phospholipid hydroperoxide glutathione peroxidase.
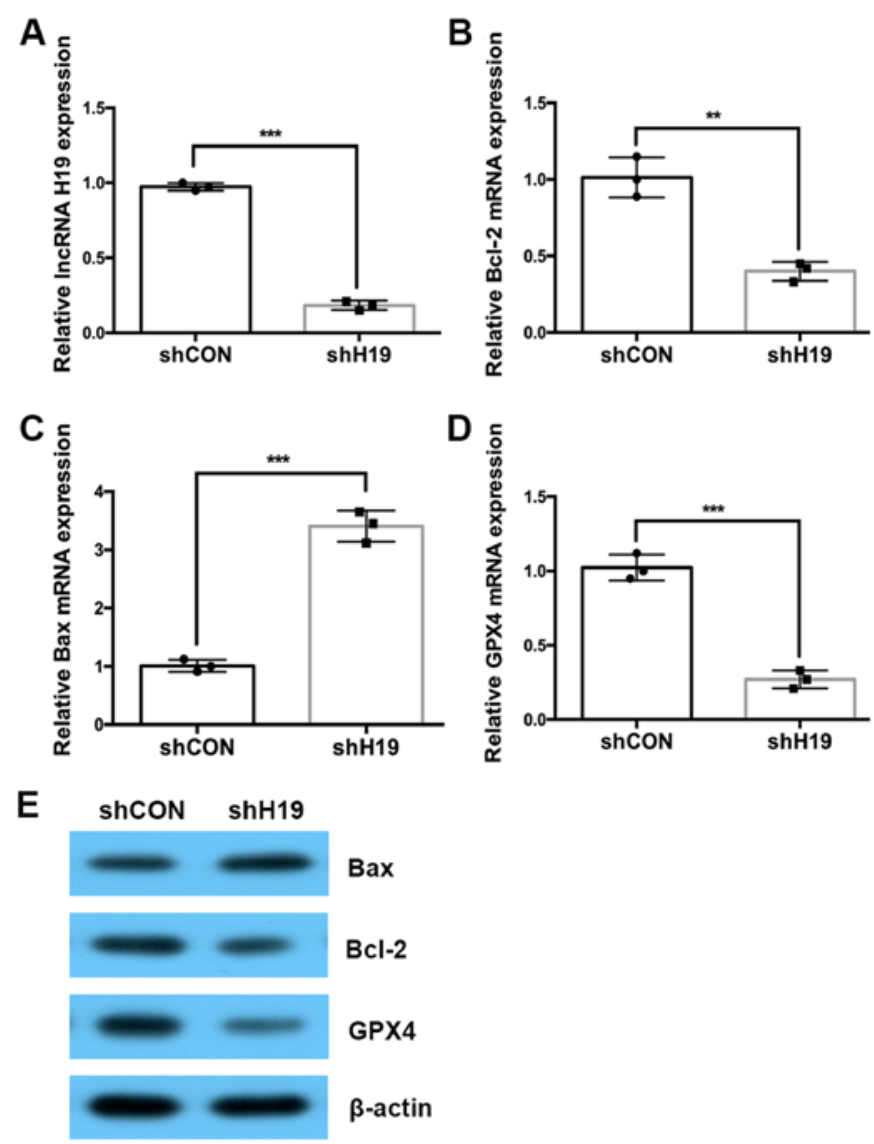

Figure 4. Silencing H19 downregulates $B c l-2$ and $G P X 4$ expression and upregulates Bax expression at both the mRNA and protein levels. (A-D) Reverse transcription-quantitative PCR was used to detect the expression of H19, Bcl-2, Bax and GPX4 in H19 silenced HTR-8 cells. (E) Western blotting was used to detect the protein expression of Bcl-2, Bax and GPX4 in the H19 silenced HTR- 8 cells. The experiments were performed three times. ${ }^{* * *} \mathrm{P}<0.01$ and ${ }^{* * * *} \mathrm{P}<0.001$ vs. control. sh, short hairpin; shNC, negative control group; shH19, H19 knockdown group; GPX4, phospholipid hydroperoxide glutathione peroxidase; IncRNA, long non-coding RNA. Circle, expression data of shCON group; square, expression data of shH19 group.

of $B c l-2$ and $G P X 4$ and increased Bax expression at both the mRNA and protein levels (Fig. 4B-E).

The viability of HTR-8 cells following H19 knockdown and apoptosis or ferroptosis inhibitor treatment was further examined. The results showed that $\mathrm{H} 19$ silencing significantly reduced the viability of HTR-8 cells, and Z-VAD-FMK (an apoptosis inhibitor) and Ferrostatin-1 (a ferroptosis inhibitor) (22) could partially rescue the decreased viability of HTR-8 cells induced by H19 knockdown (Fig. 5).

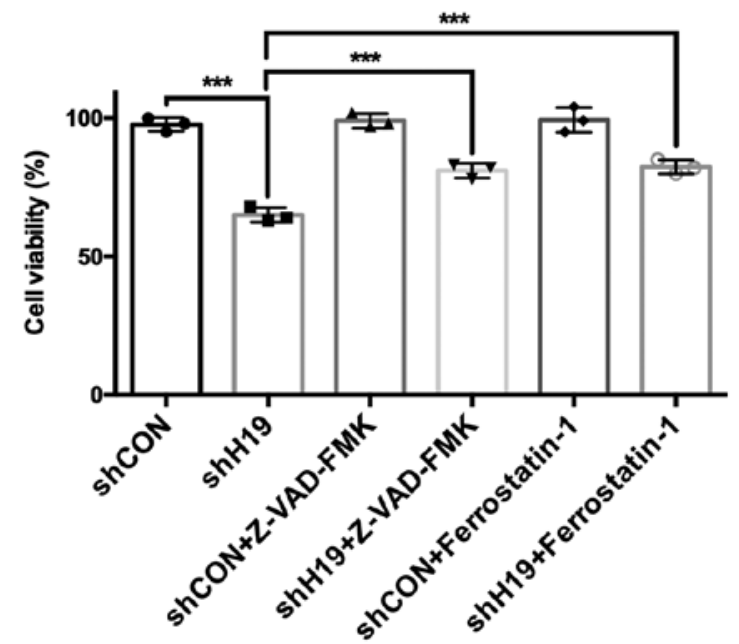

Figure 5. Apoptosis and ferroptosis inhibitors partially rescue decreased cell viability induced by H19 knockdown in HTR- 8 cells. Cell viability was detected by using a Cell Counting Kit- 8 assay. The experiments were performed three times. ${ }^{* * *} \mathrm{P}<0.001$ vs. shH19. shNC, negative control group; shH19, H19 knockdown group. Filled circle, cell viability of shCON group; square, cell viability of shH19 group; upturned triangle, cell viability of shCON+Z-VAD-FMK group; downturned triangle, cell viability of shH19+ Z-VAD-FMK group; diamond, cell viability of shCON+Ferrostatin-1 group; unfilled circle, cell viability of shH19+Ferrostatin-1 group.

\section{Discussion}

SA is the most frequently occurring pregnancy disorder and is a serious threat to women's health (23). SA has been reported to be associated with several risk factors, including endocrine disorders, anatomic deformation, immunodeficiency and chromosomal abnormalities (24-27). However, other risk factors and underlying molecular mechanisms of SA still need further investigation.

H19, a lncRNA, is involved in the pathogenesis of several diseases, including lung cancer and pre-eclampsia $(28,29)$. Although a previous study reported that the methylation of H19 differentially methylated regions is increased in the placental samples of SA cases (23), the role and underlying mechanism of H19 in SA are still largely unclear. The present study revealed that the expression of H19 was lower in SA group compared with that in control group.

Trophoblast cells participate in the formation of placenta and have important functions in embryo implantation (30). Apoptosis of trophoblast cells is involved in SA (31). The apoptotic rate and mRNA and protein expression of Fas and FasL were significantly higher in recurrent spontaneous abortion compared with the abortion group (32). Li et al (33) reported that knockdown of Storkhead-box protein 1 induced apoptosis and decreased the migration and proliferation of HTR-8/SVneo cells via mediating the PI3K/AKT signaling pathway (33). A previous study also reported that miR-184, which is highly expressed in RSA, repressed the proliferation and promoted the apoptosis of trophoblast cells by targeting WIG1 and upregulating its downstream molecule Fas (7). Dong et al (8) also found that miR-520, which is highly expressed in villi in RSA, enhanced the apoptosis of human trophoblast cells by targeting and regulating PARPI expression. Increased cyclin-dependent kinase inhibitor 1 and Bax expression was associated with increased apoptosis of cytotrophoblasts and human decidual cells in the RSA group (34). 
However, the regulatory mechanisms underlying trophoblast apoptosis are still largely unknown. The present results demonstrated that the mRNA and protein expression of $\mathrm{Bcl}-2$ was lower and the expression of Bax was higher in SA compared with control tissues. Notably, the expression of $\mathrm{Bcl}-2$ was positively correlated with $\mathrm{H} 19$ expression, and Bax expression was negatively correlated with $H 19$ levels. In vitro analysis revealed that silencing $\mathrm{H} 19$ decreased $\mathrm{Bcl}-2$ and upregulated Bax expression at both the mRNA and protein levels.

Ferroptosis is a caspase-independent, non-apoptotic and newly defined type of cell death characterized by high levels of lipid peroxidation (35). GPX4 is the key regulator of ferroptosis by controlling the activity of cyclooxygenase and lipid-modulating lipoxygenase enzymes (36). A recent study has reported that an inactivating mutation of GPX4 has a dominant-negative effect on male fertility (36). However, the roles of ferroptosis and GPX4 in SA are still unknown. The present findings reported that GPX4 expression was lower in SA group compared with that in control group and was positively correlated with $H 19$ expression. In addition, silencing H19 downregulated GPX4 expression levels. Overall, these results indicated that ferroptosis was involved in SA.

The mechanisms by which IncRNA H19 regulates $B c l-2$, Bax and GPX4 expression is still unclear. A previous study reported that $\mathrm{H} 19$ could directly binds to the miR-17-92 cluster and suppress STAT3 activity, decreasing the expression of its target genes $\mathrm{Bcl}-2$ and $\mathrm{Bcl}-2 \mathrm{Ll}$ (37). H19 is also reported to positively regulate $B c l-2$ expression by sponging miR-877-3p in myocardial ischemia/reperfusion injury (I/RI) (38). Whether $\mathrm{H} 19$ regulates $\mathrm{Bcl}-2$ by these two pathways or other pathways in SA still needs to be explored.

There are some limitations to the present study. Firstly, the regulatory association between $\mathrm{H} 19$ and $\mathrm{Bcl}-2$, Bax and GPX4 was confirmed in only one cell line. In the future, more cell lines should be used to verify the present findings. Secondly, an Annexin V/PI and lipid peroxidation assays should be used in the figure to investigate the regulatory association between H19 and apoptosis and ferroptosis.

Overall, the present study demonstrated that $H 19$ was downregulated in SA cases, and $H 19$ expression was positively correlated with $\mathrm{Bcl}-2$ and $\mathrm{GPX} 4$ levels, and negatively correlated with Bax expression. In addition, silencing H19 downregulated $\mathrm{Bcl}-2$ and $\mathrm{GPX} 4$, and upregulated $\mathrm{Bax}$ at both the mRNA and protein levels. The results of the current study may provide a novel insight for the pathogenesis for SA.

\section{Acknowledgements}

Not applicable.

\section{Funding}

The present study was funded by The Open Project Program of Yunnan Provincial Key Laboratory for Birth Defects and Genetic Diseases (grant no. 2017ZDKFKT001).

\section{Availability of data and materials}

All data generated or analyzed during this study are included in this published article.

\section{Authors' contributions}

RXB and ZYT designed the study. RXB and ZYT performed the experiments. ZYT wrote the paper. All authors read and approved the final manuscript.

\section{Ethics approval and consent to participate}

The present study was approved by The Medical Ethics Committee of the First People's Hospital of Yunnan Province (approval no. 2018-012, Kunming, China) and written informed consent was provided by each patient.

\section{Patient consent for publication}

Not applicable.

\section{Competing interests}

The authors declare that they have no competing interests.

\section{References}

1. Van Den Berg MM, Van Maarie MC, Van Wely M and Goddijn M: Genetics of early miscarriage. Biochim Biophys Acta 1822: 1951-1959, 2012

2. Tur-Torres $\mathrm{MH}$, Garrido-Gimenez $\mathrm{C}$ and Alijotas-Reig J: Genetics of recurrent miscarriage and fetal loss. Best Pract Res Clin Obstet Gynaecol 42: 11-25, 2017.

3. Regan L and Rai R: Epidemiology and the medical causes of miscarriage. Baillieres Best Pract Res Clin Obstet Gynaecol 14: 839-854, 2000.

4. Christiansen OB, Steffensen R, Nielsen HS and Varming K: Multifactorial etiology of recurrent miscarriage and its scientific and clinical implications. Gynecol Obstet Invest 66: 257-267, 2008.

5. Warren JE and Silver RM: Genetics of pregnancy loss. Clin Obstet Gynecol 51: 84-95, 2008.

6. Cinar O, Kara F and Can A: Potential role of decidual apoptosis in the pathogenesis of miscarriages. Gynecol Endocrinol 28: 382-385, 2012

7. Zhang Y, Zhou J, Li MQ, Xu J, Zhang JP and Jin LP: MicroRNA-184 promotes apoptosis of trophoblast cells via targeting WIG1 and induces early spontaneous abortion. Cell Death Dis 10: 223, 2019.

8. Dong $X$, Yang $L$ and Wang $H$ : miR-520 promotes DNA-damage-induced trophoblast cell apoptosis by targeting PARP1 in recurrent spontaneous abortion (RSA). Gynecol Endocrinol 33: 274-278, 2017.

9. Dixon SJ, Lemberg KM, Lamprecht MR, Skouta R, Zaitsev EM, Gleason CE, Patel DN, Bauer AJ, Cantley AM, Yang WS, et al: Ferroptosis: An iron-dependent form of nonapoptotic cell death. Cell 149: 1060-1072, 2012.

10. Friedmann Angeli JP, Schneider M, Proneth B, Tyurina YY, Tyurin VA, Hammond VJ, Herbach N, Aichler M, Walch A, Eggenhofer E, et al: Inactivation of the ferroptosis regulator Gpx4 triggers acute renal failure in mice. Nat Cell Biol 16: 1180-1191, 2014.

11. Linkermann A, Skouta R, Himmerkus N, Mulay SR, Dewitz C, De Zen F, Prokai A, Zuchtriegel G, Krombach F, Welz PS, et al: Synchronized renal tubular cell death involves ferroptosis. Proc Natl Acad Sci USA 111: 16836-16841, 2014.

12. Alim I, Caulfield JT, Chen Y, Swarup V, Geschwind DH, Ivanova E, Seravalli J, Ai Y, Sansing LH, Ste Marie EJ, et al: Selenium drives a transcriptional adaptive program to block ferroptosis and treat stroke. Cell 177: 1262-1279 e1225, 2019.

13. Cheng G, Song Z, Liu Y, Xiao H, Ruan H, Cao Q, Wang K, Xiao W, Xiong Z, Liu D, et al: Long noncoding RNA SNHG12 indicates the prognosis of prostate cancer and accelerates tumorigenesis via sponging miR-133b. J Cell Physiol 235: 1235-1246, 2020.

14. Guttman M and Rinn JL: Modular regulatory principles of large non-coding RNAs. Nature 482: 339-346, 2012. 
15. Matouk I, Raveh E, Ohana P, Lail RA, Gershtain E, Gilon M, De Groot N, Czerniak A and Hochberg A: The increasing complexity of the oncofetal H19 gene locus: Functional dissection and therapeutic intervention. Int J Mol Sci 14: 4298-4316, 2013.

16. Zheng ZH, Wu DM, Fan SH, Zhang ZF, Chen GQ and Lu J: Upregulation of miR-675-5p induced by lncRNA H19 was associated with tumor progression and development by targeting tumor suppressor p53 in non-small cell lung cancer. J Cell Biochem 120: 18724-18735, 2019.

17. Si H, Chen P, Li H and Wang X: Long non-coding RNA H19 regulates cell growth and metastasis via miR-138 in breast cancer. Am J Transl Res 11: 3213-3225, 2019.

18. Hu L, Zeng $\mathrm{H}$ and Liu N: Expression of H19 long non-coding RNA and ZEB1 in the trophoblast of women with spontaneous abortion. Zhong Nan Da Xue Xue Bao Yi Xue Ban 43: 179-183, 2018 (In Chinese)

19. Liu Y, Tang Y, Ye D, Ma W, Feng S, Li X, Zhou X, Chen X and Chen S: Impact of abnormal DNA methylation of imprinted loci on human spontaneous abortion. Reprod Sci 25: 131-139, 2018.

20. Livak KJ and Schmittgen TD: Analysis of relative gene expression data using real-time quantitative PCR and the 2(-Delta Delta C(T)) method. Methods 25: 402-408, 2001.

21. Xu X, Wang J, Han K, Li S, Xu F and Yang Y: Antimalarial drug mefloquine inhibits nuclear factor kappa B signaling and induces apoptosis in colorectal cancer cells. Cancer Sci 109: 1220-1229, 2018.

22. Bai T, Wang S, Zhao YP, Zhu RT, Wang WJ and Sun YL: Haloperidol, a sigma receptor 1 antagonist, promotes ferroptosis in hepatocellular carcinoma cells. Biochem Biophys Res Commun 491: 919-925, 2017.

23. Vasconcelos S, Ramalho C, Marques CJ and Doria S: Altered expression of epigenetic regulators and imprinted genes in human placenta and fetal tissues from second trimester spontaneous pregnancy losses. Epigenetics 14: 1234-1244, 2019.

24. Caseiro AL, Regalo A, Pereira E, Esteves T, Fernandes F and Carvalho J: Implication of sperm chromosomal abnormalities in recurrent abortion and multiple implantation failure. Reprod Biomed Online 31: 481-485, 2015.

25. Li TC, Makris M, Tomsu M, Tuckerman E and Laird S: Recurrent miscarriage: Aetiology, management and prognosis. Hum Reprod Update 8: 463-481, 2002.

26. Kaur R and Gupta K: Endocrine dysfunction and recurrent spontaneous abortion: An overview. Int J Appl Basic Med Res 6: 79-83, 2016.

27. Patriarca A, Piccioni V, Gigante V and Benedetto C: The use of intravenous immunoglobulin in sine causa or alloimmune recurrent spontaneous abortion (RSA). Panminerva Med 42: 193-195, 2000
28. Shu C, Yan D, Chen C, Mo Y, Wu L, Gu J, Shah NK, He J and Dong S: Metformin exhibits its therapeutic effect in the treatment of pre-eclampsia via modulating the Met/H19/miR-148a-5p/P28 and Met/H19/miR-216-3p/EBI3 signaling pathways. Int Immunopharmacol 74: 105693, 2019.

29. Liao S, Yu C, Liu H, Zhang C, Li Y and Zhong X: Long non-coding RNA H19 promotes the proliferation and invasion of lung cancer cells and regulates the expression of E-cadherin, N-cadherin, and vimentin. Onco Targets Ther 12: 4099-4107, 2019.

30. Hustin J, Jauniaux E and Schaaps JP: Histological study of the materno-embryonic interface in spontaneous abortion. Placenta 11: 477-486, 1990.

31. Xiang H, Yan H, Sun B, Feng F and Chen P: Decreased expression of long non-coding RNA SNHG7 cause recurrent spontaneous abortion through suppression proliferation and invasion of trophoblast cells via miR-34a. Am J Transl Res 11: 463-472, 2019.

32. Sun $\mathrm{Q}$ and Zhang XL: Research on apoptotic signaling pathways of recurrent spontaneous abortion caused by dysfunction of trophoblast infiltration. Eur Rev Med Pharmacol Sci 21 (Suppl 3): 12-19, 2017

33. Li Z, Zhou G, Jiang L, Xiang H and Cao Y: Effect of STOX1 on recurrent spontaneous abortion by regulating trophoblast cell proliferation and migration via the PI3K/AKT signaling pathway. J Cell Biochem 120: 8291-8299, 2018.

34. Lv X, Cai Z and Li S: Increased apoptosis rate of human decidual cells and cytotrophoblasts in patients with recurrent spontaneous abortion as a result of abnormal expression of CDKN1A and Bax. Exp Ther Med 12: 2865-2868, 2016.

35. Seiler A, Schneider M, Förster H, Roth S, Wirth EK, Culmsee C, Plesnila N, Kremmer E, Rådmark $\mathrm{O}$, Wurst W, et al: Glutathione peroxidase 4 senses and translates oxidative stress into 12/15-lipoxygenase dependent- and AIF-mediated cell death. Cell Metab 8: 237-248, 2008.

36. Ingold I, Aichler M, Yefremova E, Roveri A, Buday K, Doll S, Tasdemir A, Hoffard N, Wurst W, Walch A, et al: Expression of a catalytically inactive mutant form of glutathione peroxidase 4 (Gpx4) confers a dominant-negative effect in male fertility. J Biol Chem 290: 14668-14678, 2015.

37. Zhang A, Shang W, Nie Q, Li T and Li S: Long non-coding RNA H19 suppresses retinoblastoma progression via counteracting miR-17-92 cluster. J Cell Biochem 119: 3497-3509, 2018.

38. Li X, Luo S, Zhang J, Yuan Y, Jiang W, Zhu H, Ding X, Zhan L, Wu H, Xie Y, et al: lncRNA H19 alleviated myocardial I/RI via suppressing miR-877-3p/Bcl-2-mediated mitochondrial apoptosis. Mol Ther Nucleic Acids 17: 297-309, 2019. 